\title{
EXPLICIT COMPUTATION OF THE CHERN CHARACTER FORMS
}

\author{
LEON A. TAKHTAJAN
}

\begin{abstract}
We propose a method for explicit computation of the Chern character form of a holomorphic Hermitian vector bundle $(E, h)$ over a complex manifold $X$ in a local holomorphic frame. First, we use the descent equations arising in the double complex of $(p, q)$-forms on $X$ and find explicit degree decomposition of the Chern-Simons form $\mathrm{cs}_{k}$ associated to the Chern character form $\mathrm{ch}_{k}$ of $(E, h)$. Second, we introduce the 'ascent' equations that start from the $(2 k-1,0)$ component of $\mathrm{cs}_{k}$, and use Cholesky decomposition of the Hermitian metric $h$ to represent the Chern-Simons form, modulo $d$-exact forms, as a $\partial$-exact form. This yields a formula for the Bott-Chern form $\mathrm{bc}_{k}$ of type $(k-1, k-1)$ such that $\mathrm{ch}_{k}=\frac{\sqrt{-1}}{2 \pi} \bar{\partial} \partial \mathrm{bc}_{k}$. Explicit computation is presented for the cases $k=2$ and 3 .
\end{abstract}

\section{INTRODUCTION}

Let $V$ be a $C^{\infty}$-complex vector bundle with a connection $\nabla=d+A$ over a smooth manifold $X$. The Chern character form $\operatorname{ch}(V, \nabla)$ for the pair $(V, \nabla)$ is defined by

$$
\operatorname{ch}(V, \nabla)=\operatorname{tr}\left\{\exp \left(\frac{\sqrt{-1}}{2 \pi} \nabla^{2}\right)\right\} .
$$

Here $\nabla^{2}$ is the curvature of the connection $\nabla$, End $V$-valued 2-form on $X$, and $t r$ is the trace in the endomorphism bundle End $V$. The Chern character form is closed, $d \operatorname{ch}(V, \nabla)=0$, and its cohomology class in $H^{*}(X, \mathbb{C})$ does not depend on the choice of $\nabla$ (see, e.g., [1]).

Let $\nabla^{0}$ and $\nabla^{1}$ be two connections on $V$. In [2], S.S. Chern and J. Simons introduced secondary characteristic forms - the Chern-Simons forms $\operatorname{cs}\left(\nabla^{1}, \nabla^{0}\right)$. They are defined modulo exact forms, satisfy the equation

$$
d \operatorname{cs}\left(\nabla^{1}, \nabla^{0}\right)=\operatorname{ch}\left(V, \nabla^{1}\right)-\operatorname{ch}\left(V, \nabla^{0}\right),
$$

and enjoy a functoriality property under the pullbacks with smooth maps. When the bundle $V$ is flat, putting $\nabla^{1}=d+A$ and $\nabla^{0}=d$ and using linear homotopy $A(t)=t A$ in the Chern-Weil homotopy formula, one obtains an explicit formula for the Chern-Simons form $\operatorname{cs}(A)$ in terms of $A$.

Let $(E, h)$ be a holomorphic Hermitian vector bundle - a holomorphic vector bundle of rank $r$ over a complex manifold $X, \operatorname{dim}_{\mathbb{C}} X=n$, with a Hermitian metric $h$. Chern-Weil theory associates to any polynomial $\Phi$ on 
$\mathrm{GL}(r, \mathbb{C})$, invariant under conjugation, a differential form $\Phi(\boldsymbol{\Theta})$ on $X$. Special case of this construction is the Chern character form $\operatorname{ch}(E, h)$, defined by

$$
\operatorname{ch}(E, h)=\operatorname{tr}\left\{\exp \left(\frac{\sqrt{-1}}{2 \pi} \Theta\right)\right\}=\sum_{k=0}^{n} \operatorname{ch}_{k}(E, h) .
$$

Here $\boldsymbol{\Theta}$ is the curvature of the canonical connection $d+\boldsymbol{\theta}$ in $E$ associated with the Hermitian metric $h$. In the local holomorphic frame, $\boldsymbol{\theta}=h^{-1} \partial h$ and $\boldsymbol{\Theta}=\bar{\partial} \boldsymbol{\theta}$ (see, e.g., [1]).

Let $h_{1}$ and $h_{2}$ be two Hermitian metrics on a holomorphic vector bundle $E$ over a complex manifold $X$. In the classic paper [3], Bott and Chern showed the existence of certain secondary characteristic forms, the BottChern secondary forms bc $\left(E, h_{1}, h_{2}\right)$. They are defined modulo $\partial$ and $\bar{\partial}$-exact forms, satisfy the equation

$$
\frac{\sqrt{-1}}{2 \pi} \bar{\partial} \partial \mathrm{bc}\left(E, h_{1}, h_{2}\right)=\operatorname{ch}\left(E, h_{1}\right)-\operatorname{ch}\left(E, h_{2}\right)
$$

and enjoy the functoriality property with respect to the pullbacks by holomorphic maps. Here the Chern character forms are computed for canonical connections in $\left(E, h_{1}\right)$ and $\left(E, h_{2}\right)$. The Bott-Chern forms have been used in geometric stability $[4,5]$, in higher dimensional Arakelov geometry $[6,7]$ and in physics [8] (see also [9] for their application to differential $K$-theory).

However, it is difficult to obtain explicit formulas for the Bott-Chern forms. It is already mentioned in the remark in [3, Sect. 3] that even for a linear homotopy $h_{t}$ of Hermitian metrics, the homotopy formula in Proposition 3.15 in [3] contains the inverse metrics through $\boldsymbol{\Theta}_{t}=\bar{\partial}\left(h_{t}^{-1} \partial h_{t}\right)$ and does not allow to integrate over $t$ in a closed form. As the result, it is difficult $^{1}$ to get explicit formulas for the Bott-Chern forms in terms of Hermitian metrics $h_{1}$ and $h_{2}$ only. This problem manifests itself even for the case when $E$ is a trivial bundle with metrics $h_{1}=h$ and $h_{2}=I$, the identity matrix.

Here we show how using global coordinates on the space of Hermitian positive-definite matrices associated with the Cholesky decomposition, one can obtain explicit formulas for the Bott-Chern forms on trivial bundles. Namely, in Proposition 1 we present explicit decomposition of the ChernSimons form $\operatorname{cs}_{k}$ associated to the Chern character form $\operatorname{ch}_{k}=\operatorname{ch}_{k}(E, h)$ into $(p, q)$-degrees. It is done in Section 2 by solving the descent equations from the double complex of $(p, q)$-forms on $X$, applied to $\mathrm{ch}_{k}$. In Section 3 we introduce the 'ascent' equations that start from the $(2 k-1,0)$ component of $\mathrm{cs}_{k}$, and use Cholesky decomposition of the Hermitian metric $h$ to represent the Chern-Simons form, modulo $d$-exact forms, as a $\partial$-exact form. This yields an explicit formula for the Bott-Chern form $\mathrm{bc}_{k}$ of type $(k-1, k-1)$ such that $\operatorname{ch}_{k}=\frac{\sqrt{-1}}{2 \pi} \bar{\partial} \partial \mathrm{bc}_{k}$. It is obtained by repeatedly finding corresponding

\footnotetext{
${ }^{1}$ As was observed in [4], "One interesting feature is that we have an example of a variational problem with no simple explicit formula for the Lagrangian".
} 
$\partial$-antiderivatives and seems to be very non-local. For the case $k=2,3$ in Propositions 2 and 3 we present explicit local formulas for these forms in Cholesky coordinates. We believe that such explicit formulas exist for all $k$. In Remark 5 we prove that the form bc $_{2}$ is positive, and in Remark 7 we directly show that for bundles with upper-triangular transition functions the Euler-Lagrange functional $M_{C}(-, K)$ introduced in [4] is bounded below.

Acknowledgments. This work was done under partial support of the NSF grant DMS-1005769. I am grateful to Vamsi Pingali for stimulating discussions and suggestions, and thank Kiyoshi Igusa for his remarks.

\section{Double Descent}

2.1. Set-up. Let $h$ be Hermitian metric in rank $r$ trivial complex vector bundle over a complex manifold $X$ (i.e., in general we consider a local holomorphic frame over some open neighborhood). Put (see, e.g., [1])

$$
\boldsymbol{\theta}=h^{-1} \partial h \text { and } \boldsymbol{\Theta}=\bar{\partial} \boldsymbol{\theta} .
$$

We have the following useful formulas

$$
\partial \boldsymbol{\theta}=-\boldsymbol{\theta}^{2}, \quad \bar{\partial} \boldsymbol{\theta}=\boldsymbol{\Theta} \quad \text { and } \quad \partial \boldsymbol{\Theta}=[\boldsymbol{\Theta}, \boldsymbol{\theta}], \quad \bar{\partial} \boldsymbol{\Theta}=0,
$$

where for matrix-valued differential forms we write $A B$ instead of $A \wedge B$, etc. In particular, we have

$$
\partial \boldsymbol{\Theta}^{k}=\left[\Theta^{k}, \boldsymbol{\theta}\right] \text { and } \partial\left(\boldsymbol{\theta} \Theta^{k}\right)=-\boldsymbol{\theta} \boldsymbol{\Theta}^{k} \boldsymbol{\theta} .
$$

We have (using a 'system of units' such that $\frac{\sqrt{-1}}{2 \pi}=1$ )

$$
\operatorname{ch}_{k}(h)=\frac{1}{k !} \omega_{k, k},
$$

where

$$
\omega_{k, k}=\operatorname{tr} \Theta^{k}
$$

is $\partial$ and $\bar{\partial}$-closed real form of type $(k, k)$; here and in what follows $\omega_{p, q}$ denotes a $(p, q)$-form. It follows from Poincaré lemma that locally (i.e., on some polydisk coordinate chart of $X$ ) there are forms $\omega_{k+l, k-l-1}$ such that

$$
\begin{aligned}
\omega_{k, k} & =\bar{\partial} \omega_{k, k-1}, \\
\partial \omega_{k, k-1} & =\bar{\partial} \omega_{k+1, k-2}, \\
& \vdots \\
\partial \omega_{2 k-2,1} & =\bar{\partial} \omega_{2 k-1,0}, \\
\partial \omega_{2 k-1,0} & =0 .
\end{aligned}
$$

These descent equations ${ }^{2}$ can be written succinctly as

$$
\omega_{k, k}=(\bar{\partial}-t \partial)\left(\omega_{k, k-1}+t \omega_{k+1, k-2}+\cdots+t^{k-2} \omega_{2 k-2,1}+t^{k-1} \omega_{2 k-1,0}\right) .
$$

\footnotetext{
${ }^{2}$ Compare with the double descent in [10] and with the holomorphic descent in [8].
} 
Remark 1. Putting $t=-1$ we get

$$
\omega_{k, k}=d\left(\omega_{k, k-1}-\omega_{k+1, k-2}+\cdots+(-1)^{k-2} \omega_{2 k-2,1}+(-1)^{k-1} \omega_{2 k-1,0}\right),
$$

where $d=\partial+\bar{\partial}$. This gives an explicit decomposition of the Chern-Simons secondary form $\operatorname{cs}_{k}$ into $(p, q)$-degrees, $p+q=2 k-1$ :

$$
\operatorname{cs}_{k}=\frac{1}{k !}\left(\omega_{k, k-1}-\omega_{k+1, k-2}+\cdots+(-1)^{k-2} \omega_{2 k-2,1}+(-1)^{k-1} \omega_{2 k-1,0}\right) .
$$

It is easy to compute all these forms using (2)-(3) and equations

$$
\partial \boldsymbol{\theta}^{2}=0, \quad \bar{\partial} \boldsymbol{\theta}^{2}=[\boldsymbol{\Theta}, \boldsymbol{\theta}] .
$$

First, we observe

$$
\omega_{k, k-1}=\operatorname{tr}\left(\boldsymbol{\theta} \Theta^{k-1}\right)
$$

and state the following result.

Lemma 1. We have

$$
\partial \omega_{k, k-1}=\operatorname{tr}\left(\boldsymbol{\theta}^{2} \boldsymbol{\Theta}^{k-1}\right)=\bar{\partial} \omega_{k+1, k-2}
$$

where

$\omega_{k+1, k-2}=\frac{1}{k+1} \operatorname{tr}\left\{\boldsymbol{\theta}\left(\boldsymbol{\theta}^{2} \boldsymbol{\Theta}^{k-2}+\boldsymbol{\Theta} \boldsymbol{\theta}^{2} \boldsymbol{\Theta}^{k-3}+\cdots+\boldsymbol{\Theta}^{k-3} \boldsymbol{\theta}^{2} \boldsymbol{\Theta}+\boldsymbol{\Theta}^{k-2} \boldsymbol{\theta}^{2}\right)\right\}$.

Proof. Using (2)-(3), we get

$\partial \operatorname{tr}\left(\boldsymbol{\theta} \Theta^{k-1}\right)=\operatorname{tr}\left(-\boldsymbol{\theta}^{2} \boldsymbol{\Theta}^{k-1}-\boldsymbol{\theta}\left(\boldsymbol{\Theta}^{k-1} \boldsymbol{\theta}-\boldsymbol{\theta} \boldsymbol{\Theta}^{k-1}\right)\right)=-\operatorname{tr}\left(\boldsymbol{\theta} \boldsymbol{\Theta}^{k-1} \boldsymbol{\theta}\right)=\operatorname{tr} \boldsymbol{\theta}^{2} \boldsymbol{\Theta}^{k-1}$.

Next, using (2) and (6), we get

$$
\begin{aligned}
\bar{\partial} \sum_{i=0}^{k-2}\left(\boldsymbol{\theta} \boldsymbol{\Theta}^{i} \boldsymbol{\theta}^{2} \boldsymbol{\Theta}^{k-2-i}\right) & =\sum_{i=0}^{k-2}\left(\boldsymbol{\Theta}^{i+1} \boldsymbol{\theta}^{2} \boldsymbol{\Theta}^{k-2-i}\right)-\sum_{i=0}^{k-2}\left(\boldsymbol{\theta} \boldsymbol{\Theta}^{i}(\boldsymbol{\Theta} \boldsymbol{\theta}-\boldsymbol{\theta} \boldsymbol{\Theta}) \boldsymbol{\Theta}^{k-2-i}\right) \\
& =\sum_{i=0}^{k-2}\left(\boldsymbol{\Theta}^{i+1} \boldsymbol{\theta}^{2} \boldsymbol{\Theta}^{k-2-i}\right)+\boldsymbol{\theta}^{2} \boldsymbol{\Theta}^{k-1}-\boldsymbol{\theta} \boldsymbol{\Theta}^{k-1} \boldsymbol{\theta},
\end{aligned}
$$

since the second sum telescopes. Using the cyclic property of the trace, we get the formula for $\omega_{k+1, k-2}$.

Observe that $\omega_{k, k-1}$ is a constant term $a_{0}$ in the polynomial

$$
F_{k}(t)=\operatorname{tr}\left\{\boldsymbol{\theta}\left(\boldsymbol{\Theta}+t \boldsymbol{\theta}^{2}\right)^{k-1}\right\}=a_{0}+a_{1} t+\cdots+a_{k-1} t^{k-1}
$$

while by Lemma 1 ,

$$
\omega_{k+1, k-2}=\frac{1}{k+1} a_{1} .
$$

This suggests to consider all coefficients $a_{l}$ of $F_{k}(t)$ - differential forms of types $(k+l, k-l-1), l=0,1, \ldots, k-1$.

Lemma 2. Put $G_{k}(t)=\operatorname{tr}\left(\boldsymbol{\Theta}+t \boldsymbol{\theta}^{2}\right)^{k}$. We have

$$
\bar{\partial} F_{k}(t)-t \partial F_{k}(t)=G_{k}(t) \text {. }
$$


Proof. It follows from equations (2) and (6) that

$$
\partial\left(\boldsymbol{\Theta}+t \boldsymbol{\theta}^{2}\right)=\left[\left(\boldsymbol{\Theta}+t \boldsymbol{\theta}^{2}\right), \boldsymbol{\theta}\right] \text { and } \bar{\partial}\left(\boldsymbol{\Theta}+t \boldsymbol{\theta}^{2}\right)=t\left[\left(\boldsymbol{\Theta}+t \boldsymbol{\theta}^{2}\right), \boldsymbol{\theta}\right],
$$

which implies

$$
\partial\left(\boldsymbol{\Theta}+t \boldsymbol{\theta}^{2}\right)^{k}=\left[\left(\boldsymbol{\Theta}+t \boldsymbol{\theta}^{2}\right)^{k}, \boldsymbol{\theta}\right] \text { and } \bar{\partial}\left(\boldsymbol{\Theta}+t \boldsymbol{\theta}^{2}\right)^{k}=t\left[\left(\boldsymbol{\Theta}+t \boldsymbol{\theta}^{2}\right)^{k}, \boldsymbol{\theta}\right] .
$$

Therefore,

$$
\begin{aligned}
\partial F_{k}(t) & =\operatorname{tr}\left\{-\boldsymbol{\theta}^{2}\left(\boldsymbol{\Theta}+t \boldsymbol{\theta}^{2}\right)^{k-1}-\boldsymbol{\theta}\left(\left(\boldsymbol{\Theta}+t \boldsymbol{\theta}^{2}\right)^{k-1} \boldsymbol{\theta}-\boldsymbol{\theta}\left(\boldsymbol{\Theta}+t \boldsymbol{\theta}^{2}\right)^{k-1}\right)\right\} \\
& =\operatorname{tr}\left\{\boldsymbol{\theta}^{2}\left(\boldsymbol{\Theta}+t \boldsymbol{\theta}^{2}\right)^{k-1}\right\}
\end{aligned}
$$

and

$$
\begin{aligned}
\bar{\partial} F_{k}(t) & =\operatorname{tr}\left\{\boldsymbol{\Theta}\left(\boldsymbol{\Theta}+t \boldsymbol{\theta}^{2}\right)^{k-1}-t \boldsymbol{\theta}\left(\left(\boldsymbol{\Theta}+t \boldsymbol{\theta}^{2}\right)^{k-1} \boldsymbol{\theta}-\boldsymbol{\theta}\left(\boldsymbol{\Theta}+t \boldsymbol{\theta}^{2}\right)^{k-1}\right)\right\} \\
& =t \operatorname{tr}\left\{\boldsymbol{\theta}^{2}\left(\boldsymbol{\Theta}+t \boldsymbol{\theta}^{2}\right)^{k-1}\right\}+\operatorname{tr}\left(\boldsymbol{\Theta}+t \boldsymbol{\theta}^{2}\right)^{k},
\end{aligned}
$$

so that $(\bar{\partial}-t \partial) F_{k}(t)=G_{k}(t)$.

From here it is easy to find all descent forms $\omega_{k+l, k-l-1}$.

Proposition 1. We have

$$
\mathrm{cs}_{k}=\frac{1}{k !}\left(\omega_{k, k-1}-\omega_{k+1, k-2}+\cdots+(-1)^{k-2} \omega_{2 k-2,1}+(-1)^{k-1} \omega_{2 k-1,0}\right),
$$

where

$$
\omega_{k+l, k-l-1}=\frac{k ! l !}{(k+l) !} a_{l}, \quad l=0,1, \ldots, k-1 .
$$

In particular,

$$
\omega_{2 k-1,0}=\frac{k !(k-1) !}{(2 k-1) !} \operatorname{tr} \boldsymbol{\theta}^{2 k-1} .
$$

Proof. We observe that

$$
\frac{d G_{k}}{d t}(t)=k \operatorname{tr}\left\{\boldsymbol{\theta}^{2}\left(\boldsymbol{\Theta}+t \boldsymbol{\theta}^{2}\right)^{k-1}\right\}=k \partial F_{k}(t),
$$

so that

$$
G_{k}(t)=b_{0}+k \partial a_{0} \frac{t}{1}+k \partial a_{1} \frac{t^{2}}{2}+\cdots+k \partial a_{k-1} \frac{t^{k}}{k},
$$

where $b_{0}=\operatorname{tr} \boldsymbol{\Theta}^{k}=\omega_{k, k}$. Now it follows from Lemma 2 that

$$
\bar{\partial} a_{l}=\left(\frac{k+l}{l}\right) \partial a_{l-1}, \quad l=1, \ldots, k-1,
$$

and since $a_{0}=\omega_{k, k-1}$, we easily obtain

$$
a_{l}=\frac{(k+l) \ldots(k+1)}{l !} \omega_{k+l, k-l-1} .
$$


Thus for $k=1$ we have

$$
\omega_{1,0}=\operatorname{tr} \boldsymbol{\theta}=\partial \log \operatorname{det} h \quad \text { and } \omega_{0,0}=\log \operatorname{det} h,
$$

whereas for $k=2$

$$
\omega_{2,1}=\operatorname{tr}(\boldsymbol{\theta} \Theta) \quad \text { and } \quad \omega_{3,0}=\frac{1}{3} \operatorname{tr} \boldsymbol{\theta}^{3} .
$$

For $k=3$ we have

$$
\omega_{3,2}=\operatorname{tr}\left(\boldsymbol{\theta} \Theta^{2}\right), \quad \omega_{4,1}=\frac{1}{2} \operatorname{tr}\left(\boldsymbol{\theta}^{3} \boldsymbol{\Theta}\right) \quad \text { and } \quad \omega_{5,0}=\frac{1}{10} \operatorname{tr} \boldsymbol{\theta}^{5},
$$

and for $k=4$ from Proposition 1 we obtain

$\omega_{4,3}=\operatorname{tr}\left(\boldsymbol{\theta} \Theta^{3}\right), \quad \omega_{5,2}=\frac{1}{5} \operatorname{tr}\left(\boldsymbol{\theta}^{3} \boldsymbol{\Theta}^{2}+\boldsymbol{\theta} \boldsymbol{\Theta} \boldsymbol{\theta}^{2} \boldsymbol{\Theta}+\boldsymbol{\theta} \boldsymbol{\Theta}^{2} \boldsymbol{\theta}^{2}\right), \quad \omega_{6,1}=\frac{1}{5} \operatorname{tr}\left(\boldsymbol{\theta}^{5} \boldsymbol{\Theta}\right)$

and

$$
\omega_{7,0}=\frac{1}{35} \operatorname{tr} \boldsymbol{\theta}^{7} .
$$

Remark 2. The forms $\operatorname{tr} \boldsymbol{\theta}^{2 k-1}, k \geq 1$, where $\boldsymbol{\theta}=g^{-1} d g$ is a Maurer-Cartan form, generate the cohomology ring $H^{\bullet}(\mathrm{GL}(\infty, \mathbb{C}), \mathbb{Q})$ for the stabilized complex general linear group $\mathrm{GL}(\infty, \mathbb{C})$.

\section{Double Ascent}

3.1. Set-up. From descent equations it follows that there is a form $\omega_{2 k-2,0}$ such that

$$
\omega_{2 k-1,0}=\partial \omega_{2 k-2,0}
$$

Now going up from the bottom to the top (this explains the terminology), we get

$$
\partial\left(\omega_{2 k-2,1}+\bar{\partial} \omega_{2 k-2,0}\right)=0,
$$

so that there is a form $\omega_{2 k-3,1}$ such that

$$
\omega_{2 k-2,1}+\bar{\partial} \omega_{2 k-2,0}=\partial \omega_{2 k-3,1}
$$

Therefore

$$
\partial\left(\omega_{2 k-3,2}+\bar{\partial} \omega_{2 k-3,1}\right)=0
$$

and there is a form $\omega_{2 k-4,2}$ such that

$$
\omega_{2 k-3,2}+\bar{\partial} \omega_{2 k-3,1}=\partial \omega_{2 k-4,2}
$$

Repeating this procedure, we finally get a form $\omega_{k-1, k-1}$ such that

$$
\omega_{k, k-1}+\bar{\partial} \omega_{k, k-2}=\partial \omega_{k-1, k-1}
$$

The ascent equations can be written succinctly as

$$
k ! \operatorname{cs}_{k}=\partial \omega_{k-1, k-1}-d\left(\omega_{k, k-2}-\omega_{k+1, k-3}+\cdots+(-1)^{k} \omega_{2 k-2,0}\right) .
$$

Defining $\mathrm{CS}_{k}$ as $\mathrm{cs}_{k} \bmod \operatorname{Im} d$ (see [11]), we get

$$
\mathrm{CS}_{k}=\frac{1}{k !} \partial \omega_{k-1, k-1} \text {. }
$$


Therefore,

$$
\operatorname{ch}_{k}=\frac{1}{k !} \bar{\partial} \partial \omega_{k-1, k-1},
$$

so that $\omega_{k-1, k-1}$ is a $k$ ! times the Bott-Chern secondary form $\mathrm{bc}_{k}$ (see [3]).

Remark 3. As a corollary, we have the following version of local " $\partial \bar{\partial}$ lemma": for each form $\omega$ of type $(k, k)$ on a complex manifold $X$ satisfying $d \omega=0$ on every polydisk neighborhood $U \subset X$ there is a form $\theta_{U}$ on $U$ such that $\left.\omega\right|_{U}=\bar{\partial} \partial \theta_{U}$

Solving 'explicitly' ascent equations would give explicit local expression of the Chern character form $\mathrm{ch}_{k}$ in terms of the corresponding Bott-Chern form $\mathrm{bc}_{k}$. It is known that it is not possible to get local formulas in terms of the matrix $h$ alone. This is because each step in the ascent procedure uses Poincaré lemma which, in general, contains an integration through the homotopy formula. However, one can solve these equations explicitly by using the Cholesky decomposition!

Namely, put

$$
h=c b=b^{*} a b,
$$

where matrix $b$ is upper-triangular with 1's on the diagonal, and $a$ is diagonal with positive entries; $a_{i}$ and $b_{i j}, i=1, \ldots, r, j>i$, are global coordinates on the homogeneous space of hermitian positive-definite $r \times r$ matrices. We get

$$
\boldsymbol{\theta}=h^{-1} \partial h=b^{-1} \theta b=b^{-1}\left(\theta_{1}+\theta_{2}\right) b,
$$

where

Therefore

$$
\theta_{1}=\partial b b^{-1} \quad \text { and } \quad \theta_{2}=c^{-1} \partial c
$$

$$
\boldsymbol{\Theta}=\bar{\partial} \boldsymbol{\theta}=b^{-1}\left(\bar{\partial} \theta-\bar{\theta}_{1} \theta-\theta \bar{\theta}_{1}\right) b,
$$

where

$$
\bar{\theta}_{1}=\bar{\partial} b b^{-1} \quad \text { and } \quad \bar{\theta}_{2}=c^{-1} \bar{\partial} c .
$$

These matrix-valued 1-forms satisfy

$$
\begin{array}{cl}
\partial \theta_{1}=\theta_{1}^{2}, \quad \partial \theta_{2}=-\theta_{2}^{2}, & \bar{\partial} \bar{\theta}_{1}=\bar{\theta}_{1}^{2}, \quad \bar{\partial} \bar{\theta}_{2}=-\bar{\theta}_{2}^{2}, \\
\bar{\partial} \theta_{1}=-\partial \bar{\theta}_{1}+\theta_{1} \bar{\theta}_{1}+\bar{\theta}_{1} \theta_{1}, \quad \bar{\partial} \theta_{2}=-\partial \bar{\theta}_{2}-\theta_{2} \bar{\theta}_{2}-\bar{\theta}_{2} \theta_{2} .
\end{array}
$$

Moreover, since $\theta_{1}$ is nilpotent and

$$
\theta_{2}=a^{-1} \theta_{1}^{*} a+a^{-1} \partial a,
$$

we have important property

$$
\operatorname{tr}\left(\theta_{1}^{l_{1}} \bar{\theta}_{1}^{\bar{l}_{1}}\right)=\operatorname{tr}\left(\theta_{2}^{l_{2}} \bar{\theta}_{2}^{\bar{l}_{2}}\right)=0
$$

for all $l_{1}+\bar{l}_{1}>0$ and $l_{2}+\bar{l}_{2}>1$. We will also be using

$$
\overline{\boldsymbol{\theta}}=h^{-1} \bar{\partial} h=b^{-1} \bar{\theta} b=b^{-1}\left(\bar{\theta}_{1}+\bar{\theta}_{2}\right) b,
$$

so that

$$
\bar{\partial} \overline{\boldsymbol{\theta}}=-\overline{\boldsymbol{\theta}}^{2} \quad \text { and } \quad \boldsymbol{\Theta}=-\partial \overline{\boldsymbol{\theta}}-\boldsymbol{\theta} \overline{\boldsymbol{\theta}}-\overline{\boldsymbol{\theta}} \boldsymbol{\theta} .
$$


It turns out that in terms of $\theta_{1}, \bar{\theta}_{1}, \theta_{2}, \bar{\theta}_{2}$ and its $\partial$ and $\bar{\partial}$ differentials one can explicitly compute differential forms $\omega_{2 k-2-l, l}$ for $l=0,1, \ldots, k-1$.

Remark 4. The Cholesky decomposition is useful since by the holomorphic splitting principle (see, e.g., [12, Corollary 9.26]), for every holomorphic vector bundle $E \rightarrow X$ there exists a variety $Y$ and a flat morphism $p: Y \rightarrow$ $X$ such that the bundle $p^{*}(E)$ over $Y$ admits upper-triangular transition functions.

3.2. The case $k=2$. Start with the form $\omega_{3,0}=\frac{1}{3} \operatorname{tr} \boldsymbol{\theta}^{3}$. In terms of the Cholesky decomposition we have

$$
\omega_{3,0}=\frac{1}{3} \operatorname{tr}\left(\theta_{1}^{3}+3 \theta_{1}^{2} \theta_{2}+3 \theta_{1} \theta_{2}^{2}+\theta_{2}^{3}\right)=\operatorname{tr}\left(\theta_{1}^{2} \theta_{2}+\theta_{1} \theta_{2}^{2}\right)=\partial \operatorname{tr}\left(\theta_{1} \theta_{2}\right),
$$

so that

$$
\omega_{2,0}=\operatorname{tr}\left(\theta_{1} \theta_{2}\right)
$$

Using (15) we get

$$
\begin{aligned}
\omega_{2,1} & =\operatorname{tr}(\boldsymbol{\theta} \Theta)=-\operatorname{tr}\left(\boldsymbol{\theta}(\partial \overline{\boldsymbol{\theta}}+\boldsymbol{\theta}(\boldsymbol{\theta} \overline{\boldsymbol{\theta}}+\overline{\boldsymbol{\theta}} \boldsymbol{\theta}))=\partial \operatorname{tr}(\boldsymbol{\theta} \overline{\boldsymbol{\theta}})+\operatorname{tr}\left(\boldsymbol{\theta}^{2} \overline{\boldsymbol{\theta}}-\boldsymbol{\theta}(\boldsymbol{\theta} \overline{\boldsymbol{\theta}}+\overline{\boldsymbol{\theta}} \boldsymbol{\theta})\right)\right. \\
& =\partial \operatorname{tr}(\theta \bar{\theta})-\operatorname{tr}\left(\theta^{2} \bar{\theta}\right),
\end{aligned}
$$

and using (12) we obtain

$$
\begin{aligned}
\omega_{2,1}+\bar{\partial} \omega_{2,0}= & \partial \operatorname{tr}(\theta \bar{\theta})+\operatorname{tr}\left(-\theta^{2} \bar{\theta}+\left(\bar{\partial} \theta_{1} \theta_{2}-\theta_{1} \bar{\partial} \theta_{2}\right)\right) \\
= & \partial \operatorname{tr}(\theta \bar{\theta})+\operatorname{tr}\left(-\theta^{2} \bar{\theta}-\partial \bar{\theta}_{1} \theta_{2}+\theta_{1} \partial \bar{\theta}_{2}+\left(\theta_{1} \theta_{2}+\theta_{2} \theta_{1}\right) \bar{\theta}\right) \\
= & \partial \operatorname{tr}\left(\theta \bar{\theta}-\left(\bar{\theta}_{1} \theta_{2}-\bar{\theta}_{2} \theta_{1}\right)\right) \\
& +\operatorname{tr}\left(-\theta^{2} \bar{\theta}+\theta_{1}^{2} \bar{\theta}_{2}+\bar{\theta}_{2} \bar{\theta}_{1}^{2}+\left(\theta_{1} \theta_{2}+\theta_{2} \theta_{1}\right) \bar{\theta}\right) \\
= & \partial \operatorname{tr}\left(\theta \bar{\theta}-\left(\bar{\theta}_{1} \theta_{2}-\bar{\theta}_{2} \theta_{1}\right)\right) .
\end{aligned}
$$

Thus

$$
\omega_{1,1}=\operatorname{tr}\left(\theta \bar{\theta}-\left(\bar{\theta}_{1} \theta_{2}-\bar{\theta}_{2} \theta_{1}\right)\right)=\operatorname{tr}\left(2 \theta_{2} \bar{\theta}_{1}+\theta_{2} \bar{\theta}_{2}\right),
$$

and we obtain the following result.

Proposition 2. The second Bott-Chern form $\mathrm{bc}_{2}$ of a trivial Hermitian vector bundle $\left(\mathbb{C}^{r}, h\right)$ over a complex manifold $X$ in Cholesky coordinates $h=b^{*} a b$ is given by the formula

$$
\mathrm{bc}_{2}=\frac{1}{2} \operatorname{tr}\left(2 \theta_{2} \bar{\theta}_{1}+\theta_{2} \bar{\theta}_{2}\right) .
$$

Here $\bar{\theta}_{1}=\bar{\partial} b b^{-1}, \theta_{2}=c^{-1} \partial c$ and $\bar{\theta}_{2}=c^{-1} \bar{\partial} c$.

Remark 5. Using that $c=b^{*} a$, we obtain from (16) that

$$
\omega_{1,1}=\operatorname{tr}\left(a^{-1} \partial a \wedge a^{-1} \bar{\partial} a+2 \varphi \wedge \varphi^{*}\right),
$$

where $\varphi=a^{-1 / 2}\left(b^{*}\right)^{-1} \partial b^{*} a^{1 / 2}$, so that $\sqrt{-1} \omega_{1,1} \geq 0$. 
Remark 6. When

$$
a=\left(\begin{array}{cc}
1 & 0 \\
0 & e^{\sigma}
\end{array}\right) \quad \text { and } \quad b=\left(\begin{array}{cc}
1 & f \\
0 & 1
\end{array}\right)
$$

we get

$$
\omega_{0,0}=\sigma \quad \text { and } \quad \omega_{1,1}=\operatorname{tr}\left(\partial \sigma \wedge \bar{\partial} \sigma+2 e^{-\sigma} \partial f \wedge \bar{\partial} \bar{f}\right)
$$

so that

$$
\frac{1}{2} \bar{\partial} \partial \omega_{1,1}-\frac{1}{2}\left(\bar{\partial} \partial \omega_{0,0}\right)^{2}=\bar{\partial} \partial\left(e^{-\sigma} \partial f \wedge \bar{\partial} \bar{f}\right),
$$

in agreement with Remark 3.4 in [9].

Following Remark 4, consider rank $r$ Hermitian vector bundle $(E, h)$ with transition functions taking values in the Borel subgroup $B(r)$ of uppertriangular matrices in $\mathrm{GL}(r, \mathbb{C})$. In terms of a local trivialization of $E$ - an open cover $\left\{U_{\alpha}\right\}$ of $X$ and holomorphic transition functions $g_{\alpha \beta}: U_{\alpha} \cap U_{\beta} \rightarrow$ $B(r)$, a Hermitian metric $h$ on $E$ is given by a collection $\left\{h_{\alpha}\right\}$ of positivedefinite Hermitian matrices on $U_{\alpha}$, satisfying

$$
h_{\beta}=g_{\alpha \beta}^{*} h_{\alpha} g_{\alpha \beta} \quad \text { on } \quad U_{\alpha} \cap U_{\beta} .
$$

Denote by $\mathrm{bc}_{2 \alpha}$ the second Bott-Chern form on $U_{\alpha}$ and write $g_{\alpha \beta}=a_{\alpha \beta} b_{\alpha \beta}$, where $a_{\alpha \beta}$ are diagonal and $b_{\alpha \beta}$ are unipotent. From Proposition 2 we obtain

$$
\mathrm{bc}_{2 \beta}=\mathrm{bc}_{2 \alpha}+c_{\alpha \beta} \quad \text { on } \quad U_{\alpha} \cap U_{\beta},
$$

where

$c_{\alpha \beta}=\operatorname{tr}\left\{a_{\alpha \beta}^{-1} \partial a_{\alpha \beta} \wedge\left(\overline{a_{\alpha \beta}^{-1} \partial a_{\alpha \beta}}\right)+a_{\alpha \beta}^{-1} \partial a_{\alpha \beta} \wedge a_{\alpha}^{-1} \bar{\partial} a_{\alpha}+a_{\alpha}^{-1} \partial a_{\alpha} \wedge\left(\overline{a_{\alpha \beta}^{-1} \partial a_{\alpha \beta}}\right)\right\}$,

and depends only on $a_{\alpha \beta}$. Since $a_{\alpha \beta}$ are holomorphic, we have $\bar{\partial} \partial c_{\alpha \beta}=0$. In particular, if transition functions are unipotent, it follows from (17) that local expressions $\left\{\mathrm{bc}_{2 \alpha}\right\}$ determine a well-defined $(1,1)$-form on $X$.

Remark 7. Given two Hermitian metrics $h_{1}$ and $h_{2}$ on a holomorphic vector bundle $E$, we define a local Bott-Chern form $\mathrm{bc}_{2}\left(h_{1}, h_{2}\right)$ by

$$
\mathrm{bc}_{2}\left(h_{1}, h_{2}\right)=\mathrm{bc}_{2}\left(h_{1}\right)-\mathrm{bc}_{2}\left(h_{2}\right),
$$

where $\mathrm{bc}_{2}\left(h_{1,2}\right)$ are given in Proposition 2 with $h=h_{1,2}$. It follows from (17) that for the bundle $E$ with upper-triangular transition functions $\mathrm{bc}_{2}\left(h_{1}, h_{2}\right)$ is a well-defined $(1,1)$-form on $X$. In particular, for such bundles Proposition 2 provides an explicit formulas for the functionals $M_{\omega}(-, K)$ and $M_{C}(-, K)$ in Donaldson's paper [4], and from Remark 5 one gets that $M_{C}(-, K)$ is bounded below [4, Corollary 9].

Remark 8. Upper triangular matrices were used for the study the higher Reidemeister torsion in [13]. Though the set-up in this paper and in [13] is different, it would be interesting to compare corresponding calculations. 
3.3. The case $k=3$. Using (13) we get

$$
\begin{aligned}
\omega_{5,0} & =\frac{1}{10} \operatorname{tr} \boldsymbol{\theta}^{5}=\frac{1}{10} \operatorname{tr} \theta^{5} \\
& =\frac{1}{2} \operatorname{tr}\left(\theta_{1}^{4} \theta_{2}+\theta_{1}^{3} \theta_{2}^{2}+\theta_{1}^{2} \theta_{2} \theta_{1} \theta_{2}+\theta_{1} \theta_{2} \theta_{1} \theta_{2}^{2}+\theta_{1}^{2} \theta_{2}^{3}+\theta_{1} \theta_{2}^{4}\right) \\
& =\frac{1}{2} \partial \operatorname{tr}\left(\theta_{1}^{3} \theta_{2}+\theta_{1} \theta_{2}^{3}+\frac{1}{2}\left(\theta_{1} \theta_{2}\right)^{2}\right),
\end{aligned}
$$

so that

$$
\omega_{4,0}=\frac{1}{2} \operatorname{tr}\left(\theta_{1}^{3} \theta_{2}+\theta_{1} \theta_{2}^{3}+\frac{1}{2}\left(\theta_{1} \theta_{2}\right)^{2}\right) .
$$

We will compute $\omega_{4,1}+\bar{\partial} \omega_{4,0}$ and will find $\omega_{3,1}$ such that

$$
\omega_{4,1}+\bar{\partial} \omega_{4,0}=\partial \omega_{3,1}
$$

First using (15) we get

$$
\begin{aligned}
\omega_{4,1} & =\frac{1}{2} \operatorname{tr}\left(\boldsymbol{\theta}^{3} \boldsymbol{\Theta}\right)=-\frac{1}{2} \operatorname{tr}\left(\boldsymbol{\theta}^{3}(\partial \overline{\boldsymbol{\theta}}+\overline{\boldsymbol{\theta}} \boldsymbol{\theta}+\boldsymbol{\theta} \overline{\boldsymbol{\theta}})\right) \\
& =\frac{1}{2} \partial \operatorname{tr}\left(\boldsymbol{\theta}^{3} \overline{\boldsymbol{\theta}}\right)+\frac{1}{2} \operatorname{tr}\left(\boldsymbol{\theta}^{4} \overline{\boldsymbol{\theta}}-\boldsymbol{\theta}^{3}(\overline{\boldsymbol{\theta}} \boldsymbol{\theta}+\boldsymbol{\theta} \overline{\boldsymbol{\theta}})\right) \\
& =\frac{1}{2} \partial \operatorname{tr}\left(\theta^{3} \bar{\theta}\right)-\frac{1}{2} \operatorname{tr}\left(\theta^{4} \bar{\theta}\right) .
\end{aligned}
$$

Next, using (12) we obtain

$$
\begin{gathered}
\bar{\partial} \omega_{4,0}=\frac{1}{2} \operatorname{tr}\left(I_{1} \bar{\partial} \theta_{1}+I_{2} \bar{\partial} \theta_{2}\right) \\
=\frac{1}{2} \operatorname{tr}\left(I_{1}\left(-\partial \bar{\theta}_{1}+\theta_{1} \bar{\theta}_{1}+\bar{\theta}_{1} \theta_{1}\right)+I_{2}\left(-\partial \bar{\theta}_{2}-\theta_{2} \bar{\theta}_{2}-\bar{\theta}_{2} \theta_{2}\right)\right) \\
=\frac{1}{2} \partial \operatorname{tr}\left(I_{1} \bar{\theta}_{1}+I_{2} \bar{\theta}_{2}\right)+\frac{1}{2} \operatorname{tr}\left(\left(-\partial I_{1}+I_{1} \theta_{1}+\theta_{1} I_{1}\right) \bar{\theta}_{1}-\left(\partial I_{2}+I_{2} \theta_{2}+\theta_{2} I_{2}\right) \bar{\theta}_{2}\right),
\end{gathered}
$$

where

$$
I_{1}=\theta_{1}^{3}+\theta_{1}^{2} \theta_{2}+\theta_{2} \theta_{1}^{2}-\theta_{1} \theta_{2} \theta_{1}+\theta_{2} \theta_{1} \theta_{2}+\theta_{2}^{3}=\theta^{3}-\theta \theta_{2} \theta_{1}-\theta_{1} \theta_{2} \theta
$$

and

$$
I_{2}=-\left(\theta_{1}^{3}+\theta_{1} \theta_{2} \theta_{1}+\theta_{1} \theta_{2}^{2}-\theta_{2} \theta_{1} \theta_{2}+\theta_{2}^{2} \theta_{1}+\theta_{2}^{3}\right)=-\theta^{3}+\theta \theta_{1} \theta_{2}+\theta_{2} \theta_{1} \theta .
$$

Using identities

$$
\partial I_{1}-I_{1} \theta_{1}-\theta_{1} I_{1}=-\theta^{4} \quad \text { and } \quad \partial I_{2}+I_{2} \theta_{2}+\theta_{2} I_{2}=-\theta^{4},
$$

we get

$$
\omega_{4,1}+\bar{\partial} \omega_{4,0}=\frac{1}{2} \partial \operatorname{tr}\left(\theta^{3} \bar{\theta}+I_{1} \bar{\theta}_{1}+I_{2} \bar{\theta}_{2}\right)
$$

so that

$$
\omega_{3,1}=\frac{1}{2} \operatorname{tr}\left(\theta^{3} \bar{\theta}+I_{1} \bar{\theta}_{1}+I_{2} \bar{\theta}_{2}\right) .
$$


Equivalently,

$\omega_{3,1}=\frac{1}{2} \operatorname{tr}\left(2 \theta^{3} \bar{\theta}_{1}-\left(\theta_{1} \theta_{2}^{2}+2 \theta_{1} \theta_{2} \theta_{1}+\theta_{2}^{2} \theta_{1}\right) \bar{\theta}_{1}+\left(\theta_{1}^{2} \theta_{2}+2 \theta_{2} \theta_{1} \theta_{2}+\theta_{2} \theta_{1}^{2}\right) \bar{\theta}_{2}\right)$.

Finally, we will compute $\omega_{3,2}+\bar{\partial} \omega_{3,1}$ and find $\omega_{2,2}$ such that

$$
\omega_{3,2}+\bar{\partial} \omega_{3,1}=\partial \omega_{2,2}
$$

First, using (15) we obtain

$$
\begin{aligned}
\partial \operatorname{tr}(\boldsymbol{\theta} \Theta \overline{\boldsymbol{\theta}}) & =\operatorname{tr}\left(-\boldsymbol{\theta}^{2} \boldsymbol{\Theta} \overline{\boldsymbol{\theta}}-\boldsymbol{\theta}(\boldsymbol{\Theta} \boldsymbol{\theta}-\boldsymbol{\theta} \boldsymbol{\Theta}) \overline{\boldsymbol{\theta}}+\boldsymbol{\theta} \Theta(\boldsymbol{\Theta}+\boldsymbol{\theta} \overline{\boldsymbol{\theta}}+\overline{\boldsymbol{\theta}} \boldsymbol{\theta})\right) \\
& =\operatorname{tr}\left(\boldsymbol{\theta} \Theta^{2}+\boldsymbol{\theta}^{2} \boldsymbol{\Theta} \overline{\boldsymbol{\theta}}\right)
\end{aligned}
$$

and

$$
\begin{aligned}
\partial \operatorname{tr}(\overline{\boldsymbol{\theta}} \boldsymbol{\Theta} \boldsymbol{\theta}) & =\operatorname{tr}\left(-(\boldsymbol{\Theta}+\boldsymbol{\theta} \overline{\boldsymbol{\theta}}+\overline{\boldsymbol{\theta}} \boldsymbol{\theta}) \boldsymbol{\Theta} \boldsymbol{\theta}-\overline{\boldsymbol{\theta}}(\boldsymbol{\Theta} \boldsymbol{\theta}-\boldsymbol{\theta} \boldsymbol{\Theta}) \boldsymbol{\theta}+\overline{\boldsymbol{\theta}} \boldsymbol{\Theta} \boldsymbol{\theta}^{2}\right) \\
& =-\operatorname{tr}\left(\boldsymbol{\theta} \Theta^{2}+\overline{\boldsymbol{\theta}} \boldsymbol{\Theta} \boldsymbol{\theta}^{2}\right)
\end{aligned}
$$

so that

$$
\omega_{3,2}=\operatorname{tr}\left(\boldsymbol{\theta} \Theta^{2}\right)=\partial\left\{\frac{1}{2} \operatorname{tr}(\boldsymbol{\theta} \boldsymbol{\Theta} \overline{\boldsymbol{\theta}}-\overline{\boldsymbol{\theta}} \boldsymbol{\Theta} \boldsymbol{\theta})\right\}-\frac{1}{2} \operatorname{tr}\left(\boldsymbol{\theta}^{2} \boldsymbol{\Theta} \overline{\boldsymbol{\theta}}+\overline{\boldsymbol{\theta}} \boldsymbol{\Theta} \boldsymbol{\theta}^{2}\right) .
$$

Next, we write

$$
\omega_{3,1}=\frac{1}{2} \operatorname{tr}\left(\boldsymbol{\theta}^{3} \overline{\boldsymbol{\theta}}+\boldsymbol{I}_{1} \overline{\boldsymbol{\theta}}_{1}+\boldsymbol{I}_{2} \overline{\boldsymbol{\theta}}_{2}\right)=\omega_{3,1}^{(1)}+\omega_{3,1}^{(2)},
$$

where $\overline{\boldsymbol{\theta}}_{1}=b^{-1} \bar{\theta}_{1} b$ and $\overline{\boldsymbol{\theta}}_{2}=b^{-1} \bar{\theta}_{2} b$ and

$$
\boldsymbol{I}_{1}=\boldsymbol{\theta}^{3}-\boldsymbol{\theta} \boldsymbol{\theta}_{2} \boldsymbol{\theta}_{1}-\boldsymbol{\theta}_{1} \boldsymbol{\theta}_{2} \boldsymbol{\theta}, \quad \boldsymbol{I}_{2}=-\boldsymbol{\theta}^{3}+\boldsymbol{\theta} \boldsymbol{\theta}_{1} \boldsymbol{\theta}_{2}+\boldsymbol{\theta}_{2} \boldsymbol{\theta}_{1} \boldsymbol{\theta},
$$

where $\boldsymbol{\theta}_{1}=b^{-1} \theta_{1} b$ and $\boldsymbol{\theta}_{2}=b^{-1} \theta_{2} b$. We have

$$
\begin{aligned}
\bar{\partial} \omega_{3,1}^{(1)} & =\frac{1}{2} \bar{\partial} \operatorname{tr}\left(\boldsymbol{\theta}^{3} \overline{\boldsymbol{\theta}}\right) \\
& =\frac{1}{2} \operatorname{tr}\left(\boldsymbol{\Theta} \boldsymbol{\theta}^{2} \overline{\boldsymbol{\theta}}-\boldsymbol{\theta} \boldsymbol{\Theta} \boldsymbol{\theta} \overline{\boldsymbol{\theta}}+\boldsymbol{\theta}^{2} \boldsymbol{\Theta} \overline{\boldsymbol{\theta}}+\boldsymbol{\theta}^{3} \overline{\boldsymbol{\theta}}^{2}\right) \\
& =\frac{1}{2} \operatorname{tr}\left(\overline{\boldsymbol{\theta}} \boldsymbol{\Theta} \boldsymbol{\theta}^{2}+\boldsymbol{\theta}^{2} \boldsymbol{\Theta} \overline{\boldsymbol{\theta}}-\boldsymbol{\theta} \boldsymbol{\Theta} \boldsymbol{\theta} \overline{\boldsymbol{\theta}}+\boldsymbol{\theta}^{3} \overline{\boldsymbol{\theta}}^{2}\right),
\end{aligned}
$$

so that

$$
\omega_{3,2}+\bar{\partial} \omega_{3,1}^{(1)}=\partial\left\{\frac{1}{2} \operatorname{tr}(\boldsymbol{\theta} \boldsymbol{\Theta} \overline{\boldsymbol{\theta}}-\overline{\boldsymbol{\theta}} \boldsymbol{\Theta} \boldsymbol{\theta})\right\}+\frac{1}{2} \operatorname{tr}\left(\boldsymbol{\theta}^{3} \overline{\boldsymbol{\theta}}^{2}-\boldsymbol{\theta} \overline{\boldsymbol{\theta}} \boldsymbol{\theta} \boldsymbol{\Theta}\right) .
$$

We also have

$$
\begin{aligned}
\partial \operatorname{tr}(\boldsymbol{\theta} \overline{\boldsymbol{\theta}})^{2} & \left.\left.=2 \operatorname{tr}\left(\left(-\boldsymbol{\theta}^{2} \overline{\boldsymbol{\theta}}-\boldsymbol{\theta} \partial \overline{\boldsymbol{\theta}}\right) \boldsymbol{\theta} \overline{\boldsymbol{\theta}}\right)\right)=2 \operatorname{tr}\left(\left(-\boldsymbol{\theta}^{2} \overline{\boldsymbol{\theta}}+\boldsymbol{\theta} \boldsymbol{\Theta}+\boldsymbol{\theta} \boldsymbol{\theta} \overline{\boldsymbol{\theta}}+\boldsymbol{\theta} \overline{\boldsymbol{\theta}} \boldsymbol{\theta}\right) \boldsymbol{\theta} \overline{\boldsymbol{\theta}}\right)\right) \\
& =2 \operatorname{tr}(\boldsymbol{\theta} \boldsymbol{\Theta} \boldsymbol{\theta} \overline{\boldsymbol{\theta}}+\boldsymbol{\theta} \overline{\boldsymbol{\theta}} \boldsymbol{\theta} \boldsymbol{\theta} \overline{\boldsymbol{\theta}}),
\end{aligned}
$$

so that

$$
\operatorname{tr}(\boldsymbol{\theta} \overline{\boldsymbol{\theta}} \boldsymbol{\theta} \Theta)=\partial\left\{\frac{1}{2} \operatorname{tr}(\boldsymbol{\theta} \overline{\boldsymbol{\theta}})^{2}\right\}-\operatorname{tr}\left(\boldsymbol{\theta}^{2} \overline{\boldsymbol{\theta}} \boldsymbol{\theta} \overline{\boldsymbol{\theta}}\right)
$$


Thus we obtain

$$
\omega_{3,2}+\bar{\partial} \omega_{3,1}^{(1)}=\partial\left\{\frac{1}{2} \operatorname{tr}\left(\boldsymbol{\theta} \boldsymbol{\Theta} \overline{\boldsymbol{\theta}}-\overline{\boldsymbol{\theta}} \boldsymbol{\Theta} \boldsymbol{\theta}-\frac{1}{2}(\boldsymbol{\theta} \overline{\boldsymbol{\theta}})^{2}\right)\right\}+\frac{1}{2} \operatorname{tr}\left(\boldsymbol{\theta}^{3} \overline{\boldsymbol{\theta}}^{2}+\boldsymbol{\theta}^{2} \overline{\boldsymbol{\theta}} \boldsymbol{\theta} \overline{\boldsymbol{\theta}}\right) .
$$

So far we have not used the Cholesky decomposition and now we start using it for this case. Note that

$$
\omega_{3,2}+\bar{\partial} \omega_{3,1}^{(1)}=\partial\left\{\frac{1}{2} \operatorname{tr}\left(\boldsymbol{\theta} \Theta \overline{\boldsymbol{\theta}}-\overline{\boldsymbol{\theta}} \boldsymbol{\Theta} \boldsymbol{\theta}-\frac{1}{2}(\boldsymbol{\theta} \overline{\boldsymbol{\theta}})^{2}\right)\right\}+\frac{1}{2} \operatorname{tr}\left(\theta^{3} \bar{\theta}^{2}+\theta^{2} \bar{\theta} \theta \bar{\theta}\right)
$$

and it remains to compute

$$
\begin{aligned}
\bar{\partial} \omega_{3,1}^{(2)} & =\frac{1}{2} \bar{\partial} \operatorname{tr}\left(\boldsymbol{I}_{1} \overline{\boldsymbol{\theta}}_{1}+\boldsymbol{I}_{2} \overline{\boldsymbol{\theta}}_{2}\right)=\frac{1}{2} \bar{\partial} \operatorname{tr}\left(I_{1} \bar{\theta}_{1}+I_{2} \bar{\theta}_{2}\right) \\
& =\frac{1}{2} \operatorname{tr}\left(\bar{\partial} I_{1} \bar{\theta}_{1}+\bar{\partial} I_{2} \bar{\theta}_{2}-I_{1} \bar{\theta}_{1}^{2}+I_{2} \bar{\theta}_{2}^{2}\right) .
\end{aligned}
$$

By a straightforward computation using

$$
\bar{\partial} \theta=-\partial \bar{\theta}+\theta_{1} \bar{\theta}_{1}+\bar{\theta}_{1} \theta_{1}-\theta_{2} \bar{\theta}_{2}-\bar{\theta}_{2} \theta_{2}
$$

we get

$$
\begin{gathered}
\bar{\partial} I_{1} \bar{\theta}_{1}= \\
=\operatorname{tr}\left\{\left[\bar{\partial} \theta \theta^{2}-\theta \bar{\partial} \theta \theta+\theta^{2} \bar{\partial} \theta-\bar{\partial} \theta \theta_{2} \theta_{1}+\theta\left(\bar{\partial} \theta_{2} \theta_{1}-\theta_{2} \bar{\partial} \theta_{1}\right)-\right.\right. \\
\left.\left.-\left(\bar{\partial} \theta_{1} \theta_{2}-\theta_{1} \bar{\partial} \theta_{2}\right) \theta-\theta_{1} \theta_{2} \bar{\partial} \theta\right] \bar{\theta}_{1}\right\} \\
=\operatorname{tr}\left\{\left[-\partial \bar{\theta} \theta^{2}+\theta \partial \bar{\theta} \theta-\theta^{2} \partial \bar{\theta}+\partial \bar{\theta} \theta_{2} \theta_{1}+\theta\left(\theta_{2} \partial \bar{\theta}_{1}-\partial \bar{\theta}_{2} \theta_{1}\right)+\right.\right. \\
\left.\left.+\left(\partial \bar{\theta}_{1} \theta_{2}-\theta_{1} \partial \bar{\theta}_{2}\right) \theta+\theta_{1} \theta_{2} \partial \bar{\theta}\right] \bar{\theta}_{1}\right\}+ \\
+\operatorname{tr}\left\{\left[\left(\theta_{1} \bar{\theta}_{1}+\bar{\theta}_{1} \theta_{1}-\theta_{2} \bar{\theta}_{2}-\bar{\theta}_{2} \theta_{2}\right) \theta^{2}-\theta\left(\theta_{1} \bar{\theta}_{1}+\bar{\theta}_{1} \theta_{1}-\theta_{2} \bar{\theta}_{2}-\bar{\theta}_{2} \theta_{2}\right) \theta+\right.\right. \\
+\theta^{2}\left(\theta_{1} \bar{\theta}_{1}+\bar{\theta}_{1} \theta_{1}-\theta_{2} \bar{\theta}_{2}-\bar{\theta}_{2} \theta_{2}\right)-\left(\theta_{1} \bar{\theta}_{1}+\bar{\theta}_{1} \theta_{1}-\theta_{2} \bar{\theta}_{2}-\bar{\theta}_{2} \theta_{2}\right) \theta_{2} \theta_{1}- \\
-\theta\left(\left(\theta_{2} \bar{\theta}_{2}+\bar{\theta}_{2} \theta_{2}\right) \theta_{1}+\theta_{2}\left(\theta_{1} \bar{\theta}_{1}+\bar{\theta}_{1} \theta_{1}\right)\right)-\left(\left(\theta_{1} \bar{\theta}_{1}+\bar{\theta}_{1} \theta_{1}\right) \theta_{2}+\theta_{1}\left(\theta_{2} \bar{\theta}_{2}+\bar{\theta}_{2} \theta_{2}\right)\right) \theta- \\
\left.\left.-\theta_{1} \theta_{2}\left(\theta_{1} \bar{\theta}_{1}+\bar{\theta}_{1} \theta_{1}-\theta_{2} \bar{\theta}_{2}-\bar{\theta}_{2} \theta_{2}\right)\right] \bar{\theta}_{1}\right\}
\end{gathered}
$$

and

$$
\begin{gathered}
\bar{\partial} I_{2} \bar{\theta}_{2}= \\
=\operatorname{tr}\left\{\left[-\bar{\partial} \theta \theta^{2}+\theta \bar{\partial} \theta \theta-\theta^{2} \bar{\partial} \theta+\bar{\partial} \theta \theta_{1} \theta_{2}-\theta\left(\bar{\partial} \theta_{1} \theta_{2}-\theta_{1} \bar{\partial} \theta_{2}\right)+\right.\right. \\
\left.\left.+\left(\bar{\partial} \theta_{2} \theta_{1}-\theta_{2} \bar{\partial} \theta_{1}\right) \theta+\theta_{2} \theta_{1} \bar{\partial} \theta\right] \bar{\theta}_{2}\right\} \\
=\operatorname{tr}\left\{\left[\partial \bar{\theta} \theta^{2}-\theta \partial \bar{\theta} \theta+\theta^{2} \partial \bar{\theta}-\partial \bar{\theta} \theta_{1} \theta_{2}-\theta\left(\theta_{1} \partial \bar{\theta}_{2}-\partial \bar{\theta}_{1} \theta_{2}\right)-\right.\right. \\
\left.\left.-\left(\partial \bar{\theta}_{2} \theta_{1}-\theta_{2} \partial \bar{\theta}_{1}\right) \theta-\theta_{2} \theta_{1} \partial \bar{\theta}\right] \bar{\theta}_{2}\right\}+ \\
+\operatorname{tr}\left\{\left[-\left(\theta_{1} \bar{\theta}_{1}+\bar{\theta}_{1} \theta_{1}-\theta_{2} \bar{\theta}_{2}-\bar{\theta}_{2} \theta_{2}\right) \theta^{2}+\theta\left(\theta_{1} \bar{\theta}_{1}+\bar{\theta}_{1} \theta_{1}-\theta_{2} \bar{\theta}_{2}-\bar{\theta}_{2} \theta_{2}\right) \theta-\right.\right. \\
-\theta^{2}\left(\theta_{1} \bar{\theta}_{1}+\bar{\theta}_{1} \theta_{1}-\theta_{2} \bar{\theta}_{2}-\bar{\theta}_{2} \theta_{2}\right)+\left(\theta_{1} \bar{\theta}_{1}+\bar{\theta}_{1} \theta_{1}-\theta_{2} \bar{\theta}_{2}-\bar{\theta}_{2} \theta_{2}\right) \theta_{1} \theta_{2}- \\
-\theta\left(\theta_{1}\left(\theta_{2} \bar{\theta}_{2}+\bar{\theta}_{2} \theta_{2}\right)+\left(\theta_{1} \bar{\theta}_{1}+\bar{\theta}_{1} \theta_{1}\right) \theta_{2}\right)-\left(\theta_{2}\left(\theta_{1} \bar{\theta}_{1}+\bar{\theta}_{1} \theta_{1}\right)+\left(\theta_{2} \bar{\theta}_{2}+\bar{\theta}_{2} \theta_{2}\right) \theta_{1}\right) \theta+ \\
\left.\left.+\theta_{2} \theta_{1}\left(\theta_{1} \bar{\theta}_{1}+\bar{\theta}_{1} \theta_{1}-\theta_{2} \bar{\theta}_{2}-\bar{\theta}_{2} \theta_{2}\right)\right] \bar{\theta}_{2}\right\} .
\end{gathered}
$$


Thus we obtain

$$
\operatorname{tr}\left(\bar{\partial} I_{1} \bar{\theta}_{1}+\bar{\partial} I_{2} \bar{\theta}_{2}\right)=J_{1}+J_{2},
$$

where

$$
\begin{aligned}
J_{1}= & \operatorname{tr}\left\{-\partial \bar{\theta}_{2}\left(\theta_{1}^{2}+\theta_{2}^{2}+\theta_{1} \theta_{2}\right) \bar{\theta}_{1}+\left(\theta_{1}^{2}+\theta_{2}^{2}+\theta_{1} \theta_{2}\right) \partial \theta_{1} \bar{\theta}_{2}-\left(\theta_{1}^{2}+\theta_{2}^{2}+\theta_{2} \theta_{1}\right) \partial \bar{\theta}_{2} \bar{\theta}_{1}\right. \\
& +\partial \bar{\theta}_{1}\left(\theta_{1}^{2}+\theta_{2}^{2}+\theta_{2} \theta_{1}\right) \bar{\theta}_{2}+\partial \bar{\theta}_{1}\left(\theta_{2} \theta_{1}-\theta_{1} \theta_{2}\right) \bar{\theta}_{1}-\left(\theta_{2} \theta_{1}-\theta_{1} \theta_{2}\right) \partial \bar{\theta}_{1} \bar{\theta}_{1} \\
& +\partial \bar{\theta}_{2}\left(\theta_{2} \theta_{1}-\theta_{1} \theta_{2}\right) \bar{\theta}_{2}-\left(\theta_{2} \theta_{1}-\theta_{1} \theta_{2}\right) \partial \bar{\theta}_{2} \bar{\theta}_{2}+\theta_{2} \partial \bar{\theta}_{1} \theta_{2} \bar{\theta}_{1}+\theta_{2} \partial \bar{\theta}_{2} \theta_{2} \bar{\theta}_{1} \\
& -\theta_{1} \partial \bar{\theta}_{1} \theta_{1} \bar{\theta}_{2}-\theta_{1} \partial \bar{\theta}_{2} \theta_{1} \bar{\theta}_{2}+\theta_{1} \partial \bar{\theta}_{1} \theta_{2} \bar{\theta}_{1}+\theta_{2} \partial \bar{\theta}_{1} \theta_{1} \bar{\theta}_{1} \\
& \left.-\theta_{1} \partial \bar{\theta}_{2} \theta_{2} \bar{\theta}_{2}-\theta_{2} \partial \bar{\theta}_{2} \theta_{1} \bar{\theta}_{2}-\theta_{1} \partial \bar{\theta}_{2} \theta_{1} \bar{\theta}_{1}+\theta_{2} \partial \bar{\theta}_{1} \theta_{2} \bar{\theta}_{2}\right\}
\end{aligned}
$$

and

$$
\begin{aligned}
J_{2}= & \operatorname{tr}\left\{\left[\left(\theta_{1} \bar{\theta}_{1}+\bar{\theta}_{1} \theta_{1}-\theta_{2} \bar{\theta}_{2}-\bar{\theta}_{2} \theta_{2}\right) \theta^{2}-\theta\left(\theta_{1} \bar{\theta}_{1}+\bar{\theta}_{1} \theta_{1}-\theta_{2} \bar{\theta}_{2}-\bar{\theta}_{2} \theta_{2}\right) \theta\right.\right. \\
& \left.+\theta^{2}\left(\theta_{1} \bar{\theta}_{1}+\bar{\theta}_{1} \theta_{1}-\theta_{2} \bar{\theta}_{2}-\bar{\theta}_{2} \theta_{2}\right)\right]\left(\bar{\theta}_{1}-\bar{\theta}_{2}\right)-\left(\theta_{1} \bar{\theta}_{1}+\bar{\theta}_{1} \theta_{1}-\theta_{2} \bar{\theta}_{2}-\bar{\theta}_{2} \theta_{2}\right)\left(\theta_{2} \theta_{1} \bar{\theta}_{1}-\theta_{1} \theta_{2} \bar{\theta}_{2}\right) \\
& -\theta_{1} \theta_{2}\left(\theta_{1} \bar{\theta}_{1}+\bar{\theta}_{1} \theta_{1}-\theta_{2} \bar{\theta}_{2}-\bar{\theta}_{2} \theta_{2}\right) \bar{\theta}_{1}+\theta_{2} \theta_{1}\left(\theta_{1} \bar{\theta}_{1}+\bar{\theta}_{1} \theta_{1}-\theta_{2} \bar{\theta}_{2}-\bar{\theta}_{2} \theta_{2}\right) \bar{\theta}_{2} \\
& -\theta\left(\left(\theta_{2} \bar{\theta}_{2}+\bar{\theta}_{2} \theta_{2}\right) \theta_{1}+\theta_{2}\left(\theta_{1} \bar{\theta}_{1}+\bar{\theta}_{1} \theta_{1}\right)\right) \bar{\theta}_{1}-\theta\left(\theta_{1}\left(\theta_{2} \bar{\theta}_{2}+\bar{\theta}_{2} \theta_{2}\right)+\left(\theta_{1} \bar{\theta}_{1}+\bar{\theta}_{1} \theta_{1}\right) \theta_{2}\right) \bar{\theta}_{2} \\
& -\left(\theta_{1}\left(\theta_{2} \bar{\theta}_{2}+\bar{\theta}_{2} \theta_{2}\right)+\left(\theta_{1} \bar{\theta}_{1}+\bar{\theta}_{1} \theta_{1}\right) \theta_{2}\right) \theta \bar{\theta}_{1}-\left(\left(\theta_{2} \bar{\theta}_{2}+\bar{\theta}_{2} \theta_{2}\right) \theta_{1}+\theta_{2}\left(\theta_{1} \bar{\theta}_{1}+\bar{\theta}_{1} \theta_{1}\right) \theta \bar{\theta}_{2}\right\} .
\end{aligned}
$$

Simplifying and using the cyclic property of the trace, we get

$$
\begin{aligned}
J_{1}= & \operatorname{tr}\left\{\left(\theta_{1}^{2}+\theta_{2}^{2}+\theta_{1} \theta_{2}\right) \partial\left(\bar{\theta}_{1} \bar{\theta}_{2}\right)-\left(\theta_{1}^{2}+\theta_{2}^{2}+\theta_{2} \theta_{1}\right) \partial\left(\bar{\theta}_{2} \bar{\theta}_{1}\right)-\left(\theta_{2} \theta_{1}-\theta_{1} \theta_{2}\right) \partial\left(\bar{\theta}_{1}^{2}+\bar{\theta}_{2}^{2}\right)\right. \\
& +\left(\theta_{2} \partial \bar{\theta}_{1} \theta_{2} \bar{\theta}_{2}+\theta_{2} \bar{\theta}_{1} \theta_{2} \partial \bar{\theta}_{2}\right)-\left(\theta_{1} \partial \bar{\theta}_{1} \theta_{1} \bar{\theta}_{2}+\theta_{1} \bar{\theta}_{1} \theta_{1} \partial \bar{\theta}_{2}\right)+\left(\theta_{2} \partial \bar{\theta}_{1} \theta_{1} \bar{\theta}_{1}+\theta_{2} \bar{\theta}_{1} \theta_{1} \partial \bar{\theta}_{1}\right) \\
& \left.-\left(\theta_{2} \partial \bar{\theta}_{2} \theta_{1} \bar{\theta}_{2}+\theta_{2} \bar{\theta}_{2} \theta_{1} \partial \bar{\theta}_{2}\right)+\theta_{2} \partial \bar{\theta}_{1} \theta_{2} \bar{\theta}_{1}-\theta_{1} \partial \bar{\theta}_{2} \theta_{1} \bar{\theta}_{2}\right\} \\
= & \partial\left\{\operatorname { t r } \left(\left(\theta_{1}^{2}+\theta_{2}^{2}+\theta_{1} \theta_{2}\right) \bar{\theta}_{1} \bar{\theta}_{2}-\left(\theta_{1}^{2}+\theta_{2}^{2}+\theta_{2} \theta_{1}\right) \bar{\theta}_{2} \bar{\theta}_{1}-\left(\theta_{2} \theta_{1}-\theta_{1} \theta_{2}\right)\left(\bar{\theta}_{1}^{2}+\bar{\theta}_{2}^{2}\right)\right.\right. \\
& \left.-\theta_{2} \bar{\theta}_{1} \theta_{2} \bar{\theta}_{2}+\theta_{1} \bar{\theta}_{1} \theta_{1} \bar{\theta}_{2}-\theta_{2} \bar{\theta}_{1} \theta_{1} \bar{\theta}_{1}+\theta_{2} \bar{\theta}_{2} \theta_{1} \bar{\theta}_{2}+\frac{1}{2}\left(\left(\theta_{1} \bar{\theta}_{2}\right)^{2}-\left(\theta_{2} \bar{\theta}_{1}\right)^{2}\right)\right\} \\
& +\operatorname{tr}\left\{-\left(\theta_{1}^{2} \theta_{2}+\theta_{1} \theta_{2}^{2}\right) \bar{\theta}_{1} \bar{\theta}_{2}-\left(\theta_{2}^{2} \theta_{1}+\theta_{2} \theta_{1}^{2}\right) \bar{\theta}_{2} \bar{\theta}_{1}-\left(\theta_{1}^{2} \theta_{2}+\theta_{1} \theta_{2}^{2}+\theta_{2}^{2} \theta_{1}+\theta_{2} \theta_{1}^{2}\right)\left(\bar{\theta}_{1}^{2}+\bar{\theta}_{2}^{2}\right)\right. \\
& -\theta_{2}^{2} \bar{\theta}_{1} \theta_{2} \bar{\theta}_{2}-\theta_{2}^{2} \bar{\theta}_{2} \theta_{2} \bar{\theta}_{1}-\theta_{1}^{2} \bar{\theta}_{1} \theta_{1} \bar{\theta}_{2}-\theta_{1}^{2} \bar{\theta}_{2} \theta_{1} \bar{\theta}_{1}-\theta_{2}^{2} \bar{\theta}_{1} \theta_{1} \bar{\theta}_{1}+\theta_{1}^{2} \bar{\theta}_{1} \theta_{2} \bar{\theta}_{1} \\
& \left.+\theta_{2}^{2} \bar{\theta}_{2} \theta_{1} \bar{\theta}_{2}-\theta_{1}^{2} \bar{\theta}_{2} \theta_{2} \bar{\theta}_{2}-\theta_{1}^{2} \bar{\theta}_{2} \theta_{1} \bar{\theta}_{2}-\theta_{2}^{2} \bar{\theta}_{1} \theta_{2} \bar{\theta}_{1}\right\}=J_{11}+J_{12},
\end{aligned}
$$

where

$$
\begin{aligned}
J_{11}= & \partial\left\{\operatorname { t r } \left(\left(\theta_{1}^{2}+\theta_{2}^{2}+\theta_{1} \theta_{2}\right) \bar{\theta}_{1} \bar{\theta}_{2}-\left(\theta_{1}^{2}+\theta_{2}^{2}+\theta_{2} \theta_{1}\right) \bar{\theta}_{2} \bar{\theta}_{1}-\left(\theta_{2} \theta_{1}-\theta_{1} \theta_{2}\right)\left(\bar{\theta}_{1}^{2}+\bar{\theta}_{2}^{2}\right)\right.\right. \\
& \left.-\theta_{2} \bar{\theta}_{1} \theta_{2} \bar{\theta}_{2}+\theta_{1} \bar{\theta}_{1} \theta_{1} \bar{\theta}_{2}-\theta_{2} \bar{\theta}_{1} \theta_{1} \bar{\theta}_{1}+\theta_{2} \bar{\theta}_{2} \theta_{1} \bar{\theta}_{2}+\frac{1}{2}\left(\left(\theta_{1} \bar{\theta}_{2}\right)^{2}-\left(\theta_{2} \bar{\theta}_{1}\right)^{2}\right)\right\}
\end{aligned}
$$

and

$$
\begin{aligned}
J_{12}= & \operatorname{tr}\left\{-\left(\theta_{1}^{2} \theta_{2}+\theta_{1} \theta_{2}^{2}\right) \bar{\theta}_{1} \bar{\theta}_{2}-\left(\theta_{2}^{2} \theta_{1}+\theta_{2} \theta_{1}^{2}\right) \bar{\theta}_{2} \bar{\theta}_{1}-\left(\theta_{1}^{2} \theta_{2}+\theta_{1} \theta_{2}^{2}+\theta_{2}^{2} \theta_{1}+\theta_{2} \theta_{1}^{2}\right)\left(\bar{\theta}_{1}^{2}+\bar{\theta}_{2}^{2}\right)\right. \\
& -\theta_{2}^{2} \bar{\theta}_{1} \theta_{2} \bar{\theta}_{2}-\theta_{2}^{2} \bar{\theta}_{2} \theta_{2} \bar{\theta}_{1}-\theta_{1}^{2} \bar{\theta}_{1} \theta_{1} \bar{\theta}_{2}-\theta_{1}^{2} \bar{\theta}_{2} \theta_{1} \bar{\theta}_{1}-\theta_{2}^{2} \bar{\theta}_{1} \theta_{1} \bar{\theta}_{1}+\theta_{1}^{2} \bar{\theta}_{1} \theta_{2} \bar{\theta}_{1} \\
& \left.+\theta_{2}^{2} \bar{\theta}_{2} \theta_{1} \bar{\theta}_{2}-\theta_{1}^{2} \bar{\theta}_{2} \theta_{2} \bar{\theta}_{2}-\theta_{1}^{2} \bar{\theta}_{2} \theta_{1} \bar{\theta}_{2}-\theta_{2}^{2} \bar{\theta}_{1} \theta_{2} \bar{\theta}_{1}\right\}
\end{aligned}
$$


and

$$
\begin{aligned}
J_{2}= & \operatorname{tr}\left\{\left(\theta^{2}\left(\bar{\theta}_{1}-\bar{\theta}_{2}\right)\left(\theta_{1} \bar{\theta}_{1}-\theta_{2} \bar{\theta}_{2}\right)+\theta_{1} \theta^{2}\left(\bar{\theta}_{1}-\bar{\theta}_{2}\right) \bar{\theta}_{1}-\theta_{2} \theta^{2}\left(\bar{\theta}_{1}-\bar{\theta}_{2}\right) \bar{\theta}_{2}\right.\right. \\
& -\theta\left(\theta_{1} \bar{\theta}_{1}-\theta_{2} \bar{\theta}_{2}\right) \theta\left(\bar{\theta}_{1}-\bar{\theta}_{2}\right)-\theta_{1} \theta\left(\bar{\theta}_{1}-\bar{\theta}_{2}\right) \theta \bar{\theta}_{1}+\theta_{2} \theta\left(\bar{\theta}_{1}-\bar{\theta}_{2}\right) \theta \bar{\theta}_{2} \\
& +\theta^{2}\left(\theta_{1} \bar{\theta}_{1}-\theta_{2} \bar{\theta}_{2}\right)\left(\bar{\theta}_{1}-\bar{\theta}_{2}\right)+\theta^{2}\left(\bar{\theta}_{1} \theta_{1}-\bar{\theta}_{2} \theta_{2}\right)\left(\bar{\theta}_{1}-\bar{\theta}_{2}\right)-\theta_{2} \theta_{1} \bar{\theta}_{1} \theta_{1} \bar{\theta}_{1} \\
& -\theta_{1} \theta_{2} \theta_{1} \bar{\theta}_{1}^{2}+\theta_{2} \theta_{1} \bar{\theta}_{1} \theta_{2} \bar{\theta}_{2}+\theta_{2} \theta_{2} \theta_{1} \bar{\theta}_{1} \bar{\theta}_{2}+\theta_{1} \theta_{2} \bar{\theta}_{2} \theta_{1} \bar{\theta}_{1}+\theta_{1}^{2} \theta_{2} \bar{\theta}_{2} \bar{\theta}_{1} \\
& -\theta_{1} \theta_{2} \bar{\theta}_{2} \theta_{2} \bar{\theta}_{2}-\theta_{2} \theta_{1} \theta_{2} \bar{\theta}_{2}^{2}-\theta_{1} \theta_{2}\left(\theta_{1} \bar{\theta}_{1}-\theta_{2} \bar{\theta}_{2}\right) \bar{\theta}_{1}-\theta_{1} \theta_{2}\left(\bar{\theta}_{1} \theta_{1}-\bar{\theta}_{2} \theta_{2}\right) \bar{\theta}_{1} \\
& +\theta_{2} \theta_{1}\left(\theta_{1} \bar{\theta}_{1}-\theta_{2} \bar{\theta}_{2}\right) \bar{\theta}_{2}+\theta_{2} \theta_{1}\left(\bar{\theta}_{1} \theta_{1}-\bar{\theta}_{2} \theta_{2}\right) \bar{\theta}_{2}-\theta \theta_{2} \bar{\theta}_{1} \bar{\theta}_{1}-2 \theta_{2} \theta_{1} \bar{\theta}_{1} \theta \bar{\theta}_{2} \\
& -\theta \theta_{2} \theta_{1} \bar{\theta}_{1}^{2}-\theta \theta_{1} \bar{\theta}_{2} \bar{\theta}_{2}-\theta \theta_{1} \theta_{2} \bar{\theta}_{2}^{2}-2 \theta_{1} \theta_{2} \bar{\theta}_{2} \theta \bar{\theta}_{1}-\theta_{2} \theta \bar{\theta}_{1} \theta_{1} \bar{\theta}_{2}-\theta_{2} \theta \bar{\theta}_{1} \theta_{1} \bar{\theta}_{1} \\
& \left.-\theta_{1} \theta_{2} \theta \bar{\theta}_{1}^{2}-\theta_{1} \theta \bar{\theta}_{2} \theta_{2} \bar{\theta}_{2}-\theta_{2} \theta_{1} \theta \bar{\theta}_{2}^{2}-\theta_{1} \theta \bar{\theta}_{2} \theta_{2} \bar{\theta}_{1}\right\}
\end{aligned}
$$

Simplifying $J_{12}+J_{2}$ once again and after using numerous 'miraculous cancellations', we obtain

$$
\left.\tilde{J}_{1}+J_{2}+\operatorname{tr}\left(-I_{1} \bar{\theta}_{1}^{2}+I_{2} \bar{\theta}_{2}^{2}\right)\right)=-\operatorname{tr}\left(\theta^{3} \bar{\theta}^{2}+\theta^{2} \bar{\theta} \theta \bar{\theta}\right),
$$

so that finally

$$
\begin{aligned}
\omega_{3,2}+\bar{\partial} \omega_{3,1}= & \partial\left\{\frac { 1 } { 2 } \operatorname { t r } \left(\boldsymbol{\theta} \boldsymbol{\Theta} \overline{\boldsymbol{\theta}}-\overline{\boldsymbol{\theta}} \boldsymbol{\Theta} \boldsymbol{\theta}-\frac{1}{2}(\theta \bar{\theta})^{2}+\left(\theta_{1}^{2}+\theta_{2}^{2}+\theta_{1} \theta_{2}\right) \bar{\theta}_{1} \bar{\theta}_{2}\right.\right. \\
& -\left(\theta_{1}^{2}+\theta_{2}^{2}+\theta_{2} \theta_{1}\right) \bar{\theta}_{2} \bar{\theta}_{1}-\left(\theta_{2} \theta_{1}-\theta_{1} \theta_{2}\right)\left(\bar{\theta}_{1}^{2}+\bar{\theta}_{2}^{2}\right)-\theta_{2} \bar{\theta}_{1} \theta_{2} \bar{\theta}_{2} \\
& \left.+\theta_{1} \bar{\theta}_{1} \theta_{1} \bar{\theta}_{2}-\theta_{2} \bar{\theta}_{1} \theta_{1} \bar{\theta}_{1}+\theta_{2} \bar{\theta}_{2} \theta_{1} \bar{\theta}_{2}+\frac{1}{2}\left(\left(\theta_{1} \bar{\theta}_{2}\right)^{2}-\left(\theta_{2} \bar{\theta}_{1}\right)^{2}\right)\right\} .
\end{aligned}
$$

Thus we obtain the following result.

Proposition 3. The third Bott-Chern form $\mathrm{bc}_{3}$ of a trivial Hermitian vector bundle $\left(\mathbb{C}^{r}, h\right)$ over a complex manifold $X$ in Cholesky coordinates $h=b^{*} a b$ is given by the formula

$$
\begin{aligned}
\mathrm{bc}_{3}= & \frac{1}{12} \operatorname{tr}\left(\boldsymbol{\theta} \boldsymbol{\Theta} \overline{\boldsymbol{\theta}}-\overline{\boldsymbol{\theta}} \boldsymbol{\Theta} \boldsymbol{\theta}-\frac{1}{2}(\theta \bar{\theta})^{2}+\left(\theta_{1}^{2}+\theta_{2}^{2}+\theta_{1} \theta_{2}\right) \bar{\theta}_{1} \bar{\theta}_{2}\right. \\
& -\left(\theta_{1}^{2}+\theta_{2}^{2}+\theta_{2} \theta_{1}\right) \bar{\theta}_{2} \bar{\theta}_{1}-\left(\theta_{2} \theta_{1}-\theta_{1} \theta_{2}\right)\left(\bar{\theta}_{1}^{2}+\bar{\theta}_{2}^{2}\right)-\theta_{2} \bar{\theta}_{1} \theta_{2} \bar{\theta}_{2} \\
& +\theta_{1} \bar{\theta}_{1} \theta_{1} \bar{\theta}_{2}-\theta_{2} \bar{\theta}_{1} \theta_{1} \bar{\theta}_{1}+\theta_{2} \bar{\theta}_{2} \theta_{1} \bar{\theta}_{2}+\frac{1}{2}\left(\left(\theta_{1} \bar{\theta}_{2}\right)^{2}-\left(\theta_{2} \bar{\theta}_{1}\right)^{2}\right) .
\end{aligned}
$$

\section{REFERENCES}

[1] Phillip Griffiths and Joseph Harris, Principles of algebraic geometry, Wiley, 1978.

[2] S.S. Chern and J. Simons, Characteristic forms and geometric invariants, Ann. of Math. (2) 99, (1974), 48-69.

[3] R. Bott and S.S. Chern, Hermitian vector bundles and the equidistribution of the zeroes of their holomorphic sections, Acta Math. 114 (1965), 71-112.

[4] S. Donaldson, Anti-self dual Yang-Mills connections over complex surfaces and stable vector bundles, Proc. London Math. Soc., 50 (1985), 1-26.

[5] G. Tian, Bott-Chern forms and geometric stability, Discrete Contin. Dynam. Systems, 6 (2000), 211-220.

[6] H. Gillet and C. Soulé, Characteristic classes for algebraic vector bundles with hermitian metric, I, Ann. of Math., 131 (1990), 163-203. 
[7] H. Tamvakis, Arithmetic intersection theory on flag varieties, Math. Ann. 314 (1999), 641-665.

[8] Andrei Losev, Gregory Moore, Nikita Nekrasov, Samson Shatashvili, Chiral Lagrangians, Anomalies, Supersymmetry, and Holomorphy, Nucl.Phys. B484 (1997), 196-222.

[9] Vamsi P. Pingali and Leon A. Takhtajan, On Bott-Chern forms and their applications, Math. Ann. 360:1-2 (2014), 519-546.

[10] L.D. Faddeev and S.L. Shatashvili, Algebraic and Hamiltonian methods in the theory of non-Abelian anomalies, Theoret. and Math. Phys., 60:2 (1984), 770-778.

[11] James H. Simons and Dennis Sullivan, Structured vector bundles define differential K-theory, In: Quanta of maths, Clay Math. Proc., 11, pp. 579-599; Amer. Math. Soc., Providence, RI (2010).

[12] Claire Voisin, Hodge theory and complex algebraic geometry II, Cambridge U. Press, 2003.

[13] K. Igusa, Higher Franz-Reidemeister torsion, AMS/IP Studies in Advanced Mathematics, 31, 2002.

Department of Mathematics, Stony Brook University, Stony Brook, Ny 11794-3651, USA; Euler Mathematical Institute, Saint Petersburg, Russia 